\title{
Local Temperature of Out-of-Equilibrium Quantum Electron Systems
}

\author{
J. Meair ${ }^{1}$, J. P. Bergfield ${ }^{2}$, C. A. Stafford ${ }^{1}$, and Ph. Jacquod ${ }^{1,3}$ \\ ${ }^{1}$ Department of Physics, University of Arizona, 1118 E. 4th St., Tucson, AZ, 85721 \\ 2 Department of Chemistry, Northwestern University, 2145 Sheridan Road, Evanston, IL, 60208 \\ 3 College of Optical Sciences, University of Arizona, Tucson, AZ 85721
}

\begin{abstract}
We show how the local temperature of out-of-equilibrium, quantum electron systems can be consistently defined with the help of an external voltage and temperature probe. We determine sufficient conditions under which the temperature measured by the probe (i) is independent of details of the system-probe coupling, (ii) is equal to the temperature obtained from an independent current-noise measurement, (iii) satisfies the transitivity condition expressed by the zeroth law of thermodynamics, and (iv) is consistent with Carnot's theorem. This local temperature therefore characterizes the system in the common sense of equilibrium thermodynamics, but remains well defined even in out-of-equilibrium situations with no local equilibrium.
\end{abstract}

PACS numbers: 05.70.Ln, 73.63.-b, 85.50.Fi, 72.15.Jf

Introduction. Thermodynamics characterizes systems at equilibrium via equations of state that depend on a few macroscopic variables, in particular the temperature. The latter, not being an observable in the dynamical sense, can be defined in various ways. The zeroth law differentiates between classes of thermodynamic states with different temperatures, and an absolute temperature scale is introduced by the second law via Carnot's theorem. Maxwell relations express the temperature as derivatives of thermodynamic potentials with respect to the entropy. Fluctuation-dissipation theorems finally relate the temperature to equilibrium fluctuations of observables via associated response coefficients [1]. In equilibrium, these definitions are consistent with one another.

The framework of thermodynamics, and the concept of temperature in particular have been extended to nonequilibrium systems under the assumption of local equilibrium [2]. However, it has proven far more challenging to generalize the temperature concept to systems where the local equilibrium hypothesis does not hold [3, 4]. Without local equilibrium, different temperatures are commonly obtained by different measurement protocols [4]. The consensus is accordingly that trying to extend the concept of temperature to out-of-equilibrium thermodynamics can at best deliver an operational definition.

In this manuscript, we revisit and shed new light on this fundamental issue. We focus our investigations on coupled electric and thermal transport in quantum conductors brought out of equilibrium by voltage and temperature biases. We show that, under certain conditions which we specify, a local temperature can be consistently defined in this out-of-equilibrium system in the sense that: (i) the temperature is insensitive to details of the measurement protocol; (ii) the same temperature is given by at least two completely different measurements (in our case a direct thermal measurement and an electric noise measurement); (iii) two systems independently at equilibrium with a third one are also at equilibrium with one another; and (iv) the measured temperature is absolute in the sense of Carnot's theorem.

Our approach is inspired by the experimental thermometry technique of scanning thermal microscopy [5], whose resolution has recently been brought down to the nanometer range [6]. The system's local temperature is defined via an external local probe weakly coupled to the system via a tunnel barrier [7]. At its other end, the probe is connected to a macroscopic reservoir whose chemical potential and temperature are set such that neither electric nor heat current flow between the probe and the system. The probe is thus in local equilibrium with a system that is itself not at equilibrium. In linear response, the probe temperature guaranteeing this local equilibrium is unique, and we show that this temperature locally characterizes the system in the sense of points (i)(iv). The local temperature remains consistently defined even when there is no local equilibrium in the system itself. In particular, quantum interference effects that destroy equilibrium on scales comparable to the Fermi wavelength do not alter the consistency of our definition.

The physics of electronic transport in quantum coherent systems coupled to external probes dates back to Büttiker's work on dephasing [13]. Probes have been used to calculate local electronic distributions [14] and local spin accumulations [15] in such systems. The approach has recently been extended to probe thermometry in voltage- and/or temperature-biased structures 7 -12], with several investigations focusing on Fourier's law 7 11]. Ref. 12 investigated the probe temperature of AC driven systems in the weak-driving, low-frequency limit. Neglecting thermoelectric effects, it was found that the temperature measured by the probe is the same as the one extracted from a local fluctuation-dissipation relation. Recently, local temperature measurements were investigated in the more general case including thermoelectric effects, where a closed-form analytic expression for the temperature was found for open electrical circuits [7].

Refs. [5-12] considered the probe temperature as an operational definition of the local temperature of the 
sample, without examining whether this definition satisfies conditions obeyed by a temperature in the thermodynamic sense. Here, we fill this gap by investigating the fundamental issue of whether a local temperature can be consistently defined in quantum electron systems out of equilibrium and under what conditions this temperature is the same as that measured by an external probe.

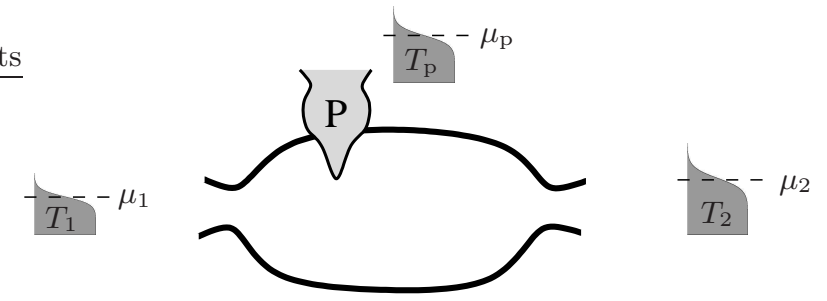

FIG. 1: Schematic of a mesoscopic conductor connected to two electron reservoirs via transport leads, and to a third reservoir via a weakly coupled probe. The chemical potentials and temperatures in all three reservoirs are indicated.

Model and scattering approach to transport. The system we investigate is sketched in Fig. 1 It is an electronic system connected to external reservoirs where electrons are thermalized and have a well defined FermiDirac distribution. One of the reservoirs is coupled to the conductor via a tunnel probe, and both its temperature $T_{\mathrm{p}}$ and chemical potential $\mu_{\mathrm{p}}$ are set such that neither electric nor heat current flow between the conductor and the probe. Thus the conductor and the probe are in local equilibrium, even though the conductor itself is not and may be traversed by heat and electric currents. We consider transport mediated solely by electrons.

We use the scattering approach to transport [13, 16, 17] which, in linear response, expresses the electrical current, $I=e I^{(0)}$, and heat current, $J=I^{(1)}$, flowing from reservoir $\alpha$ into the conductor as

$$
I_{\alpha}^{(\nu)}=\sum_{\beta}\left[\mathcal{L}_{\alpha \beta}^{(\nu, 1)}\left(\mu_{\beta}-\mu_{0}\right)+\mathcal{L}_{\alpha \beta}^{(\nu+1,1)}\left(\frac{T_{\beta}-T_{0}}{T_{0}}\right)\right] .
$$

Here, $e$ is the electron charge and $\mu_{0}$ and $T_{0}$ are the base chemical potential and temperature, which we fix at their equilibrium values. The sum over $\beta$ runs over the transport as well as the probe terminals. The linear response coefficients $\mathcal{L}$ are given by [17]

$$
\mathcal{L}_{\alpha \beta}^{(\nu, \lambda)}=\frac{1}{h} \int \mathrm{d} E\left(E-\mu_{0}\right)^{\nu}(-1)^{\lambda}\left(\partial_{E}^{\lambda} f\right) A_{\alpha \beta}(E),
$$

where $E$ is the energy, $f=\left[1+\exp \left\{\left(E-\mu_{0}\right) / k_{B} T_{0}\right\}\right]^{-1}$ is the equilibrium Fermi-Dirac distribution and $A_{\alpha \beta}(E)=$ $2 N_{\alpha}(E) \delta_{\alpha \beta}-\mathcal{T}_{\alpha \beta}(E)$, where $N_{\alpha}$ is the number of transport channels in lead $\alpha$ and $\mathcal{T}_{\alpha \beta}=\operatorname{Tr}\left[s_{\alpha \beta}^{\dagger} s_{\alpha \beta}\right]$ is given by the sub-block $s_{\alpha \beta}$ of the scattering matrix connecting lead $\beta$ to $\alpha$. The trace in this latter expression is taken over both the electron spin and the transport channels.
For the currents, only coefficients with $\lambda=1$ in Eq. (2) matter; however, those with $\lambda=2$ appear in expressions for the current noise. Charge conservation and gauge invariance are expressed mathematically by the unitarity of the scattering matrix, $\sum_{\alpha} \mathcal{L}_{\alpha \beta}^{(\nu, \lambda)}=0=\sum_{\beta} \mathcal{L}_{\alpha \beta}^{(\nu, \lambda)}$.

Probe temperature. In linear response, there is a single chemical potential $\mu_{\mathrm{p}}$ and temperature $T_{\mathrm{p}}$ ensuring $I_{\mathrm{p}}^{(0,1)}=0$. The probe temperature is

$$
\begin{aligned}
& \frac{T_{\mathrm{p}}}{T_{0}}=\kappa_{\mathrm{pp}}^{-1} \sum_{\alpha \neq \mathrm{p}} {\left[\left(\mathcal{L}_{p p}^{(0,1)} \mathcal{L}_{p \alpha}^{(1,1)}-\mathcal{L}_{p p}^{(1,1)} \mathcal{L}_{p \alpha}^{(0,1)}\right) \mu_{\alpha}\right.} \\
&\left.+\left(\mathcal{L}_{p p}^{(0,1)} \mathcal{L}_{p \alpha}^{(2,1)}-\mathcal{L}_{p p}^{(1,1)} \mathcal{L}_{p \alpha}^{(1,1)}\right) \frac{T_{\alpha}}{T_{0}}\right]
\end{aligned}
$$

with $\kappa_{p p}=\left(\mathcal{L}_{p p}^{(1,1)}\right)^{2}-\mathcal{L}_{p p}^{(0,1)} \mathcal{L}_{p p}^{(2,1)}$. Eq. (3) applies to general thermoelectric circuits (with voltage biases, temperature biases, or both) and agrees with the previous result [7] for the specific case of heat transport in an open electrical circuit. An expression similar to Eq. (3) was derived in Ref. [18]. As an example, a plot of $T_{\mathrm{p}}$ for an armchair graphene nanoribbon with a thermal bias of $\Delta T=50 \mathrm{~K}$ is shown in Fig. 2 (a).

Dependence of $T_{\mathrm{p}}$ on probe-system coupling. Let us first consider the case of single-channel probesystem coupling. For this case, it was shown in Ref. 15] that the sub-blocks $s_{\alpha p}$ (and $s_{p \alpha}$ ) of the scattering matrix factorize as $s_{\alpha p}=\gamma(E) \tilde{s}_{\alpha p}$, with the (possibly energydependent) coupling between system and probe encoded in $\gamma(E)$ only. Thus one has $\mathcal{T}_{\alpha p}(E)=|\gamma(E)|^{2} \tilde{\mathcal{T}}_{\alpha p}(E)$. Next, we rewrite Eq. (2), expanding the coefficients $A_{\alpha \beta}$ about the equilibrium chemical potential as

$$
\begin{aligned}
\mathcal{L}_{\alpha \beta}^{(\nu, 1)}= & \frac{1}{h} \int \mathrm{d} E\left(E-\mu_{0}\right)^{\nu}\left(-\partial_{E} f\right) \times\left[A_{\alpha \beta}\left(\mu_{0}\right)\right. \\
& \left.+\left(E-\mu_{0}\right)\left[\partial_{E} A_{\alpha \beta}\left(\mu_{0}\right)\right]+\mathcal{O}\left\{\left(E-\mu_{0}\right)^{2}\right\}\right] .
\end{aligned}
$$

This expansion is consistent with the Sommerfeld expansion leading, e.g. to Mott's relation for the thermopower. By symmetry, we have that the $A_{\alpha \beta}\left(\mu_{0}\right)$-term contributes when $\nu=0$ while the $\left(E-\mu_{0}\right)\left[\partial_{E} A_{\alpha \beta}\left(\mu_{0}\right)\right]$ term contributes when $\nu=1$. Setting $\beta=p$, we factorize the coefficients $A$ in the integral in Eq. (41) and write $\partial_{E} A_{\alpha p}\left(\mu_{0}\right)=\left|\gamma\left(\mu_{0}\right)\right|^{2} \partial_{E} \tilde{A}_{\alpha p}\left(\mu_{0}\right)+$ $\tilde{A}_{\alpha p}\left(\mu_{0}\right) \partial_{E}\left|\gamma\left(\mu_{0}\right)\right|^{2}$. When $\tilde{A}_{\alpha p}\left(\mu_{0}\right) \partial_{E}\left|\gamma\left(\mu_{0}\right)\right|^{2} \ll$ $\left|\gamma\left(\mu_{0}\right)\right|^{2}\left[\partial_{E} \tilde{A}_{\alpha p}\left(\mu_{0}\right)\right],\left|\gamma\left(\mu_{0}\right)\right|^{2}$ factors out of both the numerator and denominator of Eq. (3), in which case the temperature measured by the probe is independent of the strength and energy-dependence of the coupling between system and probe. Thus, as long as linear thermoelectric effects involving transmission from and to the probe are dominated by the energy-dependence of transmission coefficients inside the system [as opposed to the energy-dependence of $\gamma(E)$ ] and when the system-probe coupling proceeds via a single transport channel, $T_{\mathrm{p}}$ is independent of $\gamma(E)$. This condition is typically satisfied 



FIG. 2: (Color online) Local temperature of an armchair graphene nanoribbon probed by an atomically-sharp Pt tip scanned $3.5 \AA$ above the graphene plane. Here $\mu_{0}=-0.15 \mathrm{eV}$ from the Dirac point, and a thermal bias $\Delta T=50 \mathrm{~K}$ is applied between hot and cold electrodes forming an open electrical circuit. (a) $T_{\mathrm{p}}$ calculated from Eq. (3); (b) $T_{\mathrm{p}}^{\text {(noise) }}=T_{\mathrm{p}}(1+\chi)$ calculated from Eq. (8); (c) $T_{\mathrm{p}}$ and $T_{\mathrm{p}}^{\text {(noise) }}$ at the three points indicated in panel (a) as functions of the tip-sample coupling $\operatorname{Tr}\left\{\Gamma^{p}\right\}$, which we vary with an artificial scaling factor multiplying the tunneling-width matrix $\Gamma^{p}$. At this scan height, the tip-sample coupling is mediated by two dominant transmission channels, with an intrinsic $\operatorname{Tr}\left\{\Gamma^{p}\right\} \in[3.6 \mu \mathrm{eV}, 24 \mathrm{meV}]$ over the image.

for tunneling probes, which have transmissions that vary over an energy scale in the $\Delta \sim e V$ range. Their energydependence can therefore safely be ignored when probing nanoelectronic systems whose transmission fluctuates over a scale set by the Thouless energy $E_{\mathrm{Th}} \lesssim 10^{-1} \mathrm{eV}$ for a typical system of linear size $L \gtrsim 10 \mathrm{~nm}$.

Current noise temperature. The condition $I_{\mathrm{p}}^{(0,1)}=$ 0 leading to Eq. (3) means that time-averaged currents into the probe vanish; however, they have non-zero time- dependent fluctuations. At equilibrium, a well-known fluctuation-dissipation relation relates the zero-frequency electric current noise power to the system's equilibrium temperature, $S=4 G k_{\mathrm{B}} T_{0}[19]$, with the linear conductance $G$ of the system. We next use a Sommerfeld expansion to show that a similar fluctuation-dissipation relation exists between the probe temperature $T_{\mathrm{p}}$ and the noise of the electric current between the system and the probe.

In the scattering approach, the electric current cross correlation between terminals $\alpha$ and $\beta$ is given by [20]

$$
\begin{aligned}
\frac{S_{\alpha \beta}}{G_{0}}=\int d E & \sum_{\gamma \delta} \operatorname{Tr}\left[\mathcal{A}_{\gamma \delta}(\alpha) \mathcal{A}_{\delta \gamma}(\beta)\right] \\
& \times\left[f_{\gamma}\left(1-f_{\delta}\right)+f_{\delta}\left(1-f_{\gamma}\right)\right],
\end{aligned}
$$

where $G_{0}=e^{2} / h$ is the conductance quantum, and $\mathcal{A}_{\gamma \delta}(\alpha, E)=\mathbb{1}_{\alpha} \delta_{\alpha \gamma} \delta_{\alpha \delta}-s_{\alpha \gamma}^{\dagger}(E) s_{\alpha \delta}(E)$ with the $2 N_{\alpha} \times$ $2 N_{\alpha}$ identity matrix $\mathbb{1}_{\alpha}$. Within linear response and with Eq. (2), we obtain the current noise in the probe as

$$
S_{p p}=4 G\left(\mu_{\mathrm{p}}, T_{\mathrm{p}}\right) k_{B} T_{\mathrm{p}}^{(\text {(noise })},
$$

where

$$
\begin{aligned}
G\left(\mu_{\mathrm{p}}, T_{\mathrm{p}}\right)= & G_{0}\left[\mathcal{L}_{p p}^{(0,1)}+\mathcal{L}_{p p}^{(0,2)}\left(\mu_{\mathrm{p}}-\mu_{0}\right)\right. \\
& \left.+\left(\mathcal{L}_{p p}^{(1,2)}-\mathcal{L}_{p p}^{(0,1)}\right)\left(\frac{T_{\mathrm{p}}-T_{0}}{T_{0}}\right)\right]
\end{aligned}
$$

is the sample-to-probe conductance evaluated at the local electrochemical potential and temperature, and $T_{\mathrm{p}}^{(\text {noise })}=T_{\mathrm{p}}(1+\chi)$ with

$$
\chi=-\frac{1}{2 \mathcal{L}_{p p}^{(0,1)}} \sum_{\alpha}\left[\mathcal{L}_{p \alpha}^{(0,2)}\left(\mu_{\alpha}-\mu_{0}\right)+\mathcal{L}_{p \alpha}^{(1,2)}\left(\frac{T_{\alpha}-T_{0}}{T_{0}}\right)\right] .
$$

Eq. (6) is the Johnson-Nyquist noise for an equilibrium system with, however, $T_{\mathrm{p}}^{(\text {noise })}$ instead of $T_{\mathrm{p}}$. Clearly, when the system is at equilibrium, $T_{\mathrm{p}}^{(\text {noise })}=T_{\mathrm{p}}$, and a direct calculation using the Sommerfeld expansion shows that when the system is biased out of equilibrium, $\chi \propto$ $I_{\mathrm{p}}^{(1)}$, implying $T_{\mathrm{p}}^{(\text {noise })}=T_{\mathrm{p}}$ when $I_{\mathrm{p}}^{(1)}=0$. We conclude that the temperature $T_{\mathrm{p}}$ measured at the probe is equal to the purely electrically measured temperature $T_{\mathrm{p}}^{(\text {noise })}$ in the regime of validity of the Sommerfeld expansion.

In order to illustrate these findings, and to test their validity under somewhat more general conditions typical of a realistic tunneling probe, we calculate both $T_{\mathrm{p}}$ and $T_{\mathrm{p}}^{(\text {noise })}$ for an armchair graphene nanoribbon probed by an atomically-sharp Pt tip (see Fig. 2). The tunnel coupling is mediated by the $s, p$, and $d$ orbitals of the apex atom of the tip, leading to a coupling matrix $\Gamma^{p}$ between system's modes and probe orbitals. We found that the overlap between the $\mathrm{Pt}$ orbitals and the $\mathrm{C} \pi$-orbitals 
in graphene yields two dominant transmission channels into the tip. The connection between the eigenvalues of $\Gamma^{p}$ and the tunnel probabilities $\left|\gamma_{n}(E)\right|^{2}$ is discussed e.g. in Ref. [21]. For details of the model used for a scanning thermoelectric probe of graphene, see Refs. [7, 22].

It is apparent from Figs. 2(a) and 2(b) that the local temperatures inferred from a direct thermal measurement and from an independent current noise measurement are almost identical. Indeed, the maximum value of the discrepancy $\chi$ [cf. Eq. (8)] is just 0.0162. This agreement is remarkable, especially since there is no local equilibrium, as indicated by the short-wavelength coherent spatial oscillations of the temperature. Fig. 2(c) shows $T_{\mathrm{p}}$ and $T_{\mathrm{p}}^{(\text {noise) }}$ at three points within the sample [indicated in Fig. 2(a)] as functions of the tip-sample coupling. Despite the fact that the tip-sample coupling is effectively mediated by two transmission channels in this case, both $T_{\mathrm{p}}$ and $T_{\mathrm{p}}^{\text {(noise) }}$ are seen to be essentially independent of $\operatorname{Tr}\left\{\Gamma^{p}\right\}$ over several orders of magnitude, confirming the analytical argument given above.

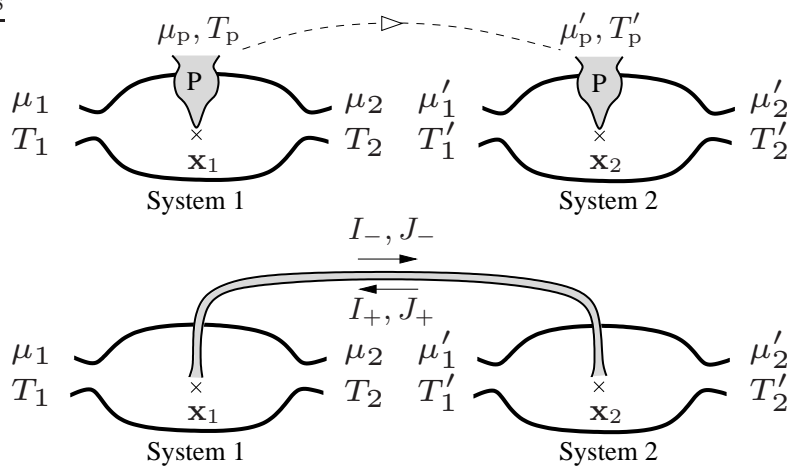

FIG. 3: Top panel: two systems sequentially probed by the same probe at local positions $\mathbf{x}_{1}$ and $\mathbf{x}_{2}$. Bottom panel: the same systems as in the top panel now connected by a transmission line locally coupled to the systems at $\mathbf{x}_{1}$ and $\mathbf{x}_{2}$.

Consistency with the zero ${ }^{\text {th }}$ law. We next use the same probe to sequentially measure the local temperature of two different, independent two-terminal systems. We assume that in the absence of bias, the two systems are at equilibrium with one another, with in particular the same equilibrium Fermi function, and that once they are biased, there is at least a pair of positions $\mathbf{x}_{1}$ on system 1 and $\mathbf{x}_{2}$ on system 2 where the probe measures the same $\mu_{\mathrm{p}}$ and $T_{\mathrm{p}}$. We then connect these two points by a transmission line with the same transparency $|\gamma(E)|^{2}$ as the probe (see Fig. 3). We assume a weak coupling $\gamma(E)$ and treat it to lowest order in perturbation theory, so that the transmission coefficients between the two systems factorize as (we use prime indices for system 2) $A_{\alpha \beta^{\prime}}=-\tilde{A}_{\alpha p}|\gamma(E)|^{2} \tilde{A}_{p \beta^{\prime}}$, in terms of the transmission coefficients $\tilde{A}$ of the disconnected systems.

The currents flowing through the neck $\left(I_{+}^{(\nu)}\right.$ from sys- tem 2 to system 1 and $I_{-}^{(\nu)}$ from 1 to 2 ) are

$$
\begin{aligned}
& I_{+}^{(\nu)}=-\frac{1}{h} \int \mathrm{d} E\left(E-\mu_{0}\right)^{\nu} \sum_{\alpha=1,2} \sum_{\beta^{\prime}=1^{\prime}, 2^{\prime}} A_{\alpha \beta^{\prime}} f_{\beta^{\prime}}, \\
& I_{-}^{(\nu)}=-\frac{1}{h} \int \mathrm{d} E\left(E-\mu_{0}\right)^{\nu} \sum_{\alpha^{\prime}=1^{\prime}, 2^{\prime}} \sum_{\beta=1,2} A_{\alpha^{\prime} \beta} f_{\beta} .
\end{aligned}
$$

Factorizing the transmission coefficients as indicated above, using the unitarity condition $\sum_{\alpha} A_{\alpha \beta}=$ $\sum_{\beta} A_{\alpha \beta}=0$, the condition of vanishing currents at the probe $I_{\mathrm{p}}^{(0,1)}=0$, and a Sommerfeld expansion, we obtain the net currents $I^{(\nu)} \equiv I_{+}^{(\nu)}-I_{-}^{(\nu)}$

$$
\left[\begin{array}{l}
I^{(0)} \\
I^{(1)}
\end{array}\right]=\left[\begin{array}{cc}
\mathcal{M} & a \partial_{E} \mathcal{M} \\
a \partial_{E} \mathcal{M} & a \mathcal{M}
\end{array}\right]\left[\begin{array}{l}
\mu_{\mathrm{p}}^{\prime}-\mu_{\mathrm{p}} \\
\frac{T_{\mathrm{p}}^{\prime}-T_{\mathrm{p}}}{T_{0}}
\end{array}\right]
$$

where $\mathcal{M}=h^{-1}|\gamma|^{2} \tilde{A}_{p p}\left(\mu_{0}\right) \tilde{A}_{p p}^{\prime}\left(\mu_{0}\right)$ and $a=$ $\left(\pi k_{B} T_{0}\right)^{2} / 3$. We see that $I^{(\nu)}=0$, i.e. the two systems, once biased, are at local equilibrium with each other when connected via points where their probe temperature and chemical potential are the same. This brings further consistency to the temperature defined by the probe, in the sense of the zeroth law of thermodynamics.

Consistency with Carnot's theorem. The junction between the system and probe can act as a heat engine when the temperature and chemical potential of the latter are biased away from their local equilibrium values $\mu_{\mathrm{p}} \rightarrow \mu_{\mathrm{p}}+\delta \mu, T_{\mathrm{p}} \rightarrow T_{\mathrm{p}}+\delta T$. The resulting flow of heat is accompanied by electrical work, and the efficiency of the engine is $\eta=-I_{\mathrm{p}}^{(0)} \delta \mu / I_{\mathrm{p}}^{(1)}$. To linear order, the currents are given by

$$
I_{\mathrm{p}}^{(\nu)}=\mathcal{L}_{p p}^{(\nu, 1)} \delta \mu+\mathcal{L}_{p p}^{(\nu+1,1)} \delta T / T_{0} .
$$

They are identical to those for a two-terminal engine in linear response, for which the maximal efficiency is

$$
\eta_{\max }=\left(\frac{\sqrt{1+\mathcal{Z} \mathcal{T}}-1}{\sqrt{1+\mathcal{Z} \mathcal{T}}+1}\right) \frac{|\delta T|}{T_{0}},
$$

with a dimensionless figure of merit $\mathcal{Z} \mathcal{T}^{-1}=$ $\mathcal{L}_{p p}^{(0,1)} \mathcal{L}_{p p}^{(2,1)} /\left(\mathcal{L}_{p p}^{(1,1)}\right)^{2}-1$. We see that, aside from linear transport coefficients, $\eta_{\max }$ depends on the ratio of the temperatures of the system and probe only, and therefore defines an absolute temperature scale in the sense of Carnot's theorem.

Conclusion. We have shown that defining the local temperature of a system out of equilibrium via an external thermal probe is consistent with both the zeroth and second laws of thermodynamics, as well as a fluctuationdissipation theorem. Moreover, the temperature is independent of the probe-sample coupling over a wide range of conditions within the tunneling regime. Importantly, 
our findings hold even when the system is far from any local equilibrium due for instance to quantum interference effects as illustrated in Fig. 2

JPB was supported by the Non-Equilibrium Energy Research Center (NERC), an Energy Frontier Research Center funded by DOE-BES under Award de-sc0000989.

CAS was supported by DOE-BES de-sc0006699.

[1] M. Kardar, Statistical Physics of Particles, Cambridge University Press (New York, 2007).

[2] S.R. de Groot and P. Mazur, Non-Equilibrium Thermodynamics, North-Holland (Amsterdam, 1962).

[3] L.F. Cugliandolo, J. Phys. A: Math. Theor. 44, 483001 (2011).

[4] J. Casas-Vásquez and D. Jou, Rep. Prog. Phys. 66, 1937 (2003).

[5] A. Majumdar, Annu. Rev. Mater. Sci. 29, 505 (1999).

[6] K. Kim, W. Jeong, W. Lee, and P. Reddy, ACS Nano 6, 4248 (2012).

[7] J.P. Bergfield, S.M. Story, R.C. Stafford, and C.A. Stafford, ACS Nano 7, 4429 (2013).

[8] P.A. Jacquet, J. Stat. Phys. 134, 709 (2009); P.A. Jacquet and C.-A. Pillet, Phys. Rev. B 85, 125120 (2012).

[9] Y. Dubi and M. Di Ventra, Nano Letters 9, 97 (2009).

[10] Y. Dubi and M. Di Ventra, Phys. Rev. E 79, 042101 (2009).

[11] Y. Dubi and M. Di Ventra, Phys. Rev. B 79, 115415 (2009).

[12] A. Caso, L. Arrachea, and G.S. Lozano, Eur. Phys. J. B 85, 266 (2012).

[13] M. Büttiker, Phys. Rev. B 33, 3020 (1986).

[14] T. Gramespacher and M. Büttiker, Phys. Rev. B 56, 13026 (1997).

[15] Ph. Jacquod, Nanotechnology 21, 274006 (2010).

[16] Y. Imry, in Directions in Condensed Matter Physics, G. Grinstein and G. Mazenko eds., World Scientific (Singapore, 1986).

[17] P. N. Butcher, J. Phys.: Condens. Matter 2, 4869 (1990).

[18] D. Sánchez and L. Serra, Phys. Rev. B 84, 201307 (2011).

[19] J.B. Johnson, Phys. Rev. 32, 91 (1928); H. Nyquist, Phys. Rev. 32, 110 (1928).

[20] Ya.M. Blanter and M. Buttiker, Phys. Rep. 336, 1 (2000).

[21] C.W.J. Beenakker, Rev. Mod. Phys. 69, 731 (1997).

[22] J.P. Bergfield, M.A. Ratner, C.A. Stafford, and M. Di Ventra, arXiv:1305.6602. 